ÉGYPTE monde arabe

\section{Égypte/Monde arabe}

30-31 | 1997

Les visions de l'Occident dans le monde arabe

\title{
L'univers en partages : altérité et voyage
}

\section{Alain Roussillon}

\section{(2) OpenEdition}

Journals

Édition électronique

URL : https://journals.openedition.org/ema/1596

DOI : 10.4000/ema.1596

ISSN : 2090-7273

\section{Éditeur}

CEDEJ - Centre d'études et de documentation économiques juridiques et sociales

\section{Édition imprimée}

Date de publication : 30 septembre 1997

Pagination : 32-65

ISSN : 1110-5097

\section{Référence électronique}

Alain Roussillon, «L'univers en partages : altérité et voyage », Égypte/Monde arabe [En ligne], 30-31 | 1997, mis en ligne le 08 juillet 2008, consulté le 07 juillet 2022. URL : http://journals.openedition.org/ ema/1596 ; DOI : https://doi.org/10.4000/ema.1596

Ce document a été généré automatiquement le 7 juillet 2022.

Tous droits réservés 


\title{
L'univers en partages : altérité et voyage
}

\author{
Alain Roussillon
}

\section{Le voyageur et son double : voyage vécu, voyage narré}

1 C'est presque un lieu commun de la littérature de voyage que de rappeler que le voyage est vécu deux fois, voire trois ${ }^{1}$. Voyage "réel », espace-temps des possibles, qui se joue à l'interface entre un système de perceptions totalisé en la personne du voyageur et de l'image de Soi que l'Autre lui renvoie. Voyage narré, où s'engage l'identité sociale du voyageur et la « commande sociale » dont sa rédaction fait l'objet : non pas tant ce que le voyageur admet de vivre dans l'éloignement de sa propre cité - et du contrôle que celle-ci exerce sur lui - que ce qu'il croit bon et utile d'en livrer au lecteur. Voyage reçu par un voyageur « virtuel », le lecteur, par quoi se constitue une «intertextualité » du voyage qui participe de la mise en représentation globale de l'altérité en contribuant singulièrement à diversifier la perception de celle-ci. À chacun de ces niveaux, ce qui se joue c'est le mode de présence du voyageur à son voyage, mode de présence dont je fais l'hypothèse qu'il est possible de mettre en évidence une corrélation significative entre celui-ci et la façon dont s'opère, dans la matérialité des textes, les partages entre identité et altérité en même temps que se mettent en place des régimes d'interactions possibles ou admissibles, ou à l'inverse d'exclusion, entre l'une et l'autre.

2 Pour ce qui est de la façon de dire « je » ou «nous » dans la relation du voyage, on peut identifier deux «tensions » contradictoires, qui passent entre les différents voyages, mais aussi, et peut-être surtout, au cœur de chaque voyage : d'une part, ce que je désignerai comme l'absence - relative, sans quoi il n'y a plus de rihla - du voyageur à son voyage: la personne même de celui-ci - c'est-à-dire l'individualité qui en vit les différents moments - tend à s'effacer derrière la fonction du voyage, soit que celle-ci lui confère une certaine qualité d'officialité ou de représentation - la rihla diplomatique -, soit que la dimension pédagogique - le voyage d'étude - ou missionnaire - le voyage militant - envahisse l'ensemble de l'espace de la rédaction. À ce pôle, le réfèrent 
collectif d'identification du voyageur - le Nous auquel il appartient, quelle que soit, par ailleurs, la façon dont celui-ci s'énonce - est pleinement activé et encode les formulations et les jugements possibles du voyageur dans les termes étroits des catégories de la reconnaissance et de l'exclusion identitaires. L'hypothèse est ici que l'absence du voyageur à son voyage, compensée par la présence massive du Nous auquel s'adresse le récit, favorise la multiplication des stéréotypes et la maximisation des différences. À l'inverse, une présence accrue, individualisée, volontaire pourrait-on dire, du voyageur à son voyage favorise, sinon nécessairement la levée des « défenses identitaires » - elle peut aboutir, on le verra, à leur exacerbation -, du moins une saisie différenciée (ce qui ne signifie pas dégagée des stéréotypes), interactive pourrait-on dire, de l'Autre.

\section{Le voyageur collectif : le voyage comme fonction}

3 Dans la courte rihla de l'ambassadeur marocain Idrîs al-'Amrâwî (1989 et 1992) à Paris, en 1860, rihla qui pourrait apparaitre comme l'une des plus « identitaires » et des plus " réfractaires ", autrement que dans ses aspects techniques ou matériels, au " génie » propre de la société française, on peut trouver une illustration significative de la façon paradoxale dont peuvent jouer ces deux logiques au sein d'un même récit pour produire deux « images» radicalement opposées de la société française dont, sauf à supposer stupide ou incohérent le narrateur - et son lecteur -, il nous faut tenter de dégager le principe de compatibilité. Avec 'Amrâwî, on est à un pôle d'officialisation maximum, avec la figure de [émissaire: le voyageur parle au nom d'un «Nous» qui s'incarne en la personne du commanditaire du voyage, le Sultan lui-même, dont l'ombre portée l'accompagne à chaque pas de sa mission. On est bien, avec 'Amrâwî, au pays des Kuffâr «chrétiens » ou «Francs " dont l'existence même, au voisinage de l'Empire, constitue une menace pour le Sultan et les musulmans. Un pays magnifique et prospère, il est vrai, mais dont il déplore qu'il soit habité par de tels infidèles, rustres et grossiers, qui

ne savent pas jouir convenablement de ces beautés, ni venir s'asseoir au bord de ce fleuve pour passer des moments agréables, ni parcourir à cheval ces prairies... Ils passent plutôt comme des démons, des possédés, montés sur des chars de fer et de feu, qui déchirent le paysage. Et lorsque, lassés, ils recherchent quelque repos et veulent profiter des agréments de la région, c'est pour entrer dans des pièces d'auberges inconfortables et boire des verres remplis d'une liqueur amère. Puis ils sortent de ces auberges en roulant comme des porcs, en sautant comme des singes, en brayant comme des ânes; leurs visages sont déformés par la manière dont ils sont rasés, et leurs propos ne sont que sifflements, grognements et éclats de rire.

Ce passage vaut d'être cité pour la façon dont s'y imbriquent les deux thématiques : on aura compris que ces «chars de fer et de feu » sont le chemin de fer auquel il vient tout juste de consacrer quatre pages émerveillées où sont décrits par le menu mérites et fonctionnement. De la même façon, il explique quelques paragraphes plus loin que ce qui fait la beauté même des paysages français, c'est leur aspect «travaillé » et la façon dont ils sont aménagés par leurs habitants. De la même façon, sa dénonciation en termes qui, s'ils ne sont pas encore "convenus ", n'en appartiennent pas moins à des registres universels, de l'immoralité des femmes françaises et de la démission des hommes est immédiatement suivie d'un passage où il souligne la parfaite moralité et la correction des transactions économiques, notant qu'il est 
une chose avérée que personne parmi eux n'accepte de pot-de-vin ni de cadeau, ni ne cherché à acquérir une marchandise quelconque à un prix inférieur au prix ordinaire, ni à obtenir un prix supérieur pour une vente, ne serait-ce que d'un drachme.

5 Au risque du paradoxe, on peut formuler deux propositions pour servir de "protocole de lecture " à la rihla de 'Amrâwî et aux textes du même acabit. D'une part, c'est la nécessité de nommer globalement l'Autre, face au Soi, qui tend à convoquer dans l'écriture les représentations les plus négatives, articulées en termes religieuxidentitaires: les dénonciations les plus «collectives» de l'Altérité, celles le plus clairement exprimées dans les catégories du «Nous», sont énoncées en termes d'indignation personnelle - façon de donner acte au mandataire ou aux destinataires de la rihla que le voyageur n'a pas été gagné à l'altérité.

6 'Amrâwî explique ainsi à son lecteur que

nous - la délégation marocaine - étions un objet d'étonnement pour les gens qui nous recevaient car ils nous voyaient demeurer chez nous la plupart du temps alors que les voitures étaient tenues à notre disposition devant le portail de notre résidence à toute heure du jour et de la nuit.

7 Comme s'il ne voyait rien et ne voulait rien voir d'inutile à sa mission, déplorant le temps et la fatigue dépensés à visiter Versailles et manifestant son incompréhension et sa réprobation à propos du théatre ou de cette manie française de collectionner toutes espèces de choses inutiles, par exemple les animaux, vivants ou morts, ou les objets anciens. Symétriquement, 'Amrâwî n'est jamais aussi " personnel », aussi présent à son voyage, que lorsqu'il suspend son jugement: ainsi note-t-il, sans autre forme de réprobation, que

la terre des Français est connue chez les chrétiens pour l'abondance et la qualité de ses vins, que l'on exporte jusqu'à des contrées lointaines, ajoutant qu'il a lui-même lu dans des gazettes à ce sujet des articles où l'on tirait fierté de cela vis-à-vis des Anglais, et où l'on disait qu'en vendant à ces derniers le vin sorti des vignes de France, on les mettait en infériorité puisqu'on prenait leur argent et leur raison en sus.

Plus loin, il relève le rôle des religieuses dans les soins aux malades de l'Hôtel des Invalides, notant que

les femmes qui s'occupent d'eux portent un habit particulier et l'on nous dit qu'elles étaient des religieuses, c'est-à-dire qu'elles avaient renoncé au mariage et s'étaient consacré au service de ces hommes, par volonté de se rapprocher de Dieu, selon leur vision des choses.

Comme si, dès lors qu'il s'agissait de rendre compte pour elles-mêmes des performances de la société française ou des personnalités singulières qui s'y rencontrent, l'exclusive religieuse était en quelque sorte levée ou suspendue et l'altérité pouvait apparaître dans ce qui fait sa positivité. On peut ne pas partager le jugement de Yadh Ben Achour sur I. AI-'Amrâwî (1992), voyageur " collectif », et par là " authentique », pour qui cette authenticité consisterait à « construire le monde dans l'innocence primordiale, en se noyant peut-être dans un abîme d'erreur, mais loin de la mauvaise foi », et qui n'aurait été intéressé, à la différence d'un Tahtâwî, plus cultivé et plus politique, « que par les décors extérieurs, les nouveautés bien visibles, les figures, les gens et les paroles ». Mais n'est-ce pas pratiquer une sorte d'exotisme à rebours que de s'étonner de l'étonnement du voyageur devant des inventions aussi étonnantes que le train, l'imprimerie ou le télégraphe ? Et, à tout prendre, la description qu'en donne l'ambassadeur marocain est aussi singulièrement exacte, pratique, opérationnelle, pourrait-on dire, propre à faire comprendre de quoi il retourne à ses compatriotes, tout comme sa façon de calculer les distances en journées de cheval ou la capacité des 
wagons de chemin de fer en charges de chameaux. De la même façon, on peut seulement choisir de considérer comme triviales les observations du voyageur quant aux manières françaises de vivre en société : la spécialisation de l'usage des pièces dans les habitations, le fait que dans les familles aisées maris et femmes aient chacun leur chambre, la régularité des horaires des repas et le fait qu'on n'oblige personne à se resservir, ou encore la spécialisation de la droite et de la gauche sur la voie publique, le fait que, dans les jardins, les arbres ne donnent pas de fruits ou la passion des Français pour les objets anciens sont certes des "détails " qui sont inscrits dans la vision du monde « communautaire » du voyageur, mais ils désignent aussi une sorte de ligne de fuite de la modernité en marche et le "cachet " propre que lui imprime tel ou tel peuple, en l'occurrence les Français. On peut voir là, comme en creux, l'identification d'une culture dont l'idée s'impose qu'elle peut être saisie par la formule de ses différences.

On peut identifier d'autres formes de dépersonnalisation du mode de présence du voyageur à son voyage, répondant à une commande sociale forte même si moins formalisée, et dont on peut montrer qu'elles produisent les mêmes effets de "stéréotypisation » et de globalisation du «Soi » et de l'altérité. Ainsi Duriyya Shafîq (s.d.) l'une des principales figures du féminisme égyptien, qui entame, au début des années cinquante, un périple à travers le monde pour défendre, dans une série de conférences le plus souvent organisées à l'initiative des ambassades égyptiennes dans les pays où elle passe, la cause de l'Egypte et des femmes égyptiennes. Dans l'ordre du récit, l'essentiel de sa rihla consiste en l'énumération d'une succession de palaces - les plus luxueux, comme il convient à la dignité de la représentante des femmes d'Égypte à quoi se résume dans la plupart des cas, avec la mention de quelques icônes, la description des villes visitées. En fait, révocation de chaque pays tend à se limiter à l'évaluation de la condition qui y est faite aux femmes et aux droits qu'elles y ont conquis, droits qui relèvent d'un universel en marche et qui ne sont, nulle part, susceptibles d'entrer en contradiction avec l'identité "authentique» des peuples visités, réalisation féminine du « Nous » dont $\mathrm{D}$. Shafîq se fait l'ambassadrice :

L'islam a toujours constitué le flambeau de tous ceux à qui il a été donné de jouer un rôle dans les mouvements féministes (ou féminins: nisâ'iya) dans le monde, et le principe de l'égalité entre l'homme et la femme dans l'islam fait partie des principes célébrés par le poète Shelley et par de nombreux autres poètes et écrivains. Comment s'expliquer, dans ces conditions, que la femme ne jouisse pas jusqu'à aujourd'hui, dans les pays musulmans, des droits que lui confère sa religion de tolérance et de générosité, qui a proclamé l'égalité entre les musulmans des centaines d'années avant que $n$ 'apparaisse le premier mouvement [féministe] en Europe.

Elle-même n'apparaît - ne se met en scène - que dans des situations " édifiantes »celles qui manifestent la reconnaissance des droits déjà conquis par les femmes en général, et la femme égyptienne en particulier, et celles qui la font témoin des progrès qui restent à accomplir - et dans les anecdotes qu'elle multiplie à propos des incidents, parfois les plus insignifiants, de son parcours, où les états d'âme qu'elle livre à son lecteur ne visent pas tant à singulariser celui-ci qu'à illustrer les situations dans lesquelles une femme voyageuse est susceptible de se trouver. Les conférences qu'elle prononce sont invariablement triomphales et suscitent toujours le plus large écho, tout comme les «personnalités féminines les plus connues dans le monde pour leur combat en faveur du droit des femmes » qu'elle rencontre sont toutes « exemplaires » et leur parcours toujours « édifiant »... Édifiantes encore, ses propres attitudes, par exemple, au moment d'entamer son périple, « ses sentiments en tant que mère » dont le coeur se 
brise en regardant ses deux filles venues lui dire au revoir en pensant aux maladies ou aux accidents qui pourraient leur arriver :

J'étais sur le point de pleurer, mais Dieu - loué soit Son nom - m'inspira le courage de retenir mes larmes et dissipa le désespoir qui était sur le point de me submerger pour la première fois de mon existence.

Dans le même registre militant, on peut évoquer les «six jours au Japon » d'Abu Bakr al-Qâdirî (1982) l'une des principales figures du parti marocain de l'Istiqlâl, à l'occasion de la tenue à Tokyo, en juin 1981, d'une réunion mondiale de l'Organisation de la conférence islamique. Le voyage est ici celui d'un missionnaire, qui communie avec les représentants des croyants du monde entier dans la propagation de la $D a^{\prime} w a$ islamique dans un pays présenté comme scientifiquement et technologiquement avancé et prêt à recevoir ce message, mais à qui ses « circonstances particulières » (dhurûfkhâssa) n'en ont pas encore donné l'occasion, un pays dont la population en a assez de l'idolâtrie "sấ'imû min al-wathaniyya " - et aspire à une foi " qui élève ses membres et leur procure la sérénité qui rend la vie heureuse et incite à des actions qui apaisent la conscience ». L'ensemble de la narration tourne autour de la mise en scène du succès de la Conférence à faire passer le message de l'islam auprès de ces "musulmans virtuels » que sont les Japonais, succès dont atteste l'apparition dans les travées de la conférence d'un groupe de musulmanes japonaises voilées «telles des anges du Seigneur nimbés de blancheur ». Les Japonais eux-mêmes ne se manifestent dans le récit qu'en tant que groupe bienveillant à l'égard des croyants réunis sur leur sol, à la fois sujet de modernité et d'authenticité :

Le Japonais a, aux heures de travail, une pensée scientifique semblable à celle de l'Européen, et après, les heures de travail une pensée authentique (asîl), fièrement attachée à ses valeurs, ses traditions et sa civilisation, dont même les danses folkloriques, quoique mixtes, inspirent au spectateur le sentiment que la dignité (wiqâr) ef la pudeur (hishma) s'imposent aux musiciens comme au public.

13 Il va de soi que l'auteur ne mentionne pas les geishas, comme le font les voyageurs plus «personnels». Plus loin, la seule individualité japonaise qui émerge dans le texte est celle du président de là communauté musulmane japonaise,

cet homme dont je continue à me demander comment il a pu avoir accès aux vérités de l'islam, percer le secret de sa législation, se laisser guider par son intentionnalité ultime au point de manifester un tel enthousiasme à leur endroit, d'en prendre la défense et de prêcher leur vérité. Je dois à la vérité, ajoute notre missionnaire, que je n'ai pas de réponse à ces questions, faute de renseignements personnels suffisants sur cet homme, ajoutant que même si tout semble confirmer son zèle, sa communication au congrès islamique ne traduisait pas, de sa part, une juste perception de la profondeur de la vérité de l'islam.

On peut évoquer, pour conclure sur ce point, une figure paradoxale qui confirme cette formule de la dépersonnalisation du mode de présence du voyageur à son voyage tout en en inversant significativement les termes et les enjeux: celle du «touriste professionnel ", auteur de relations de voyage à vocation pédagogique, vademecum pour ceux qui se risqueraient sur leurs traces ou substitut au voyage effectif pour ceux dont la curiosité pour le vaste monde se satisfait du «tourisme en chambre ». Une figure paradoxale, dans la mesure où la vocation de ces voyages est, explicitement, d'élargir le champ d'expérience du voyageur, et où les auteurs de ces rihlâty multiplient à l'envi les anecdotes et l'expression de jugements personnels sur les choses et les gens. Mais ce que montrent ces textes, c'est à quel point l'institution du «je» (du «nous») est, au premier chef, un effet d'écriture et d'encodage, en même temps que les voies et les registres selon lesquels progresse la "culture partagée ». D'une part, leur caractère 
pédagogique, la fiabilité des renseignements qu'ils fournissent et leur crédibilité même dépendent en quelque sorte de l'effacement du voyageur lui-même, ou mieux, de sa «standardisation » : l'auteur est ici, pour les avoir pratiqués, celui qui sait ce qu'est un bon hôtel ou un bon restaurant, ce qu'est un moyen de transport commode ou un paysage qui vaut le détour. Les hôtels de Tiflis sont convenables, note Rashshâd Bey (s.d.) président en retraite d'un tribunal du Caire,

mais le meilleur d'entre eux est l'Hôtel de l'Orient, en face du palais du gouverneur, qui ne le cède en rien aux plus grands palaces d'Europe par la propreté, l'organisation ou la qualité de son restaurant. Il suffit de dire qu'il est dirigé par un Français, à la différence des autres hôtel du sud du Caucase qui sont pour la plupart entre les mains de Géorgiens ou d'Arméniens et qui ressemblent aux hôtels arméniens du Caire.

Qu'est-ce à dire, si ce n'est que l'auteur a les mêmes exigences et les mêmes critères d'appréciation qu'un touriste européen et que les itinéraires qu'il suggère ou les étapes qu'il recommande seront "sans surprise", propres à satisfaire des exigences proprement universelles, indépendantes de l'identité propre du voyageur dont on peut considérer que, quelle que soit son origine, il appréciera de voyager dans les meilleures conditions et d'en avoir pour son argent. D'autre part, la contrepartie de cet effacement ou de cette universalisation du voyageur est le plein déploiement de la spécificité de l'autre, qui semble dès lors devoir occuper tout l'espace de la relation :

Chaque nation (umma) a ses coutumes nationales ('âdât ahliyya), ses coutumes religieuses, ses procédures officielles et règlements administratifs, que l'étranger (al-gharîb) se doit de respecter, même s'ils divergent des coutumes et des traditions de son propre peuple (qawmihî). De la même façon, chaque individu a son caractère et son tempérament qu'autrui (al-ghayr) doit éviter de heurter, même si ceux-ci ne correspondent pas à son propre caractère et à son propre tempérament.

16 À l'universalisation de la figure du voyageur correspond, dans cette formulation, la relativisation absolue de l'altérité : d'une part, on est toujours le gharîb de quelqu'un d'autre, identité universelle négative, pourrait-on dire, qui est le lot de chaque touriste dès lors qu'il s'éloigne de sa patrie d'origine; de l'autre, le modus vivendi proposé pour les relations entre les cultures peut être assimilé à celui qui devrait régir les relations entre les individus (al-ghayi) - respect des différences et évitement des conflits. Une fois ceci posé, l'« étrangéité » du monde et des sociétés pourra être déployée dans l'ordre du récit comme mise en scène de l'infinie diversité des réalisations de l'altérité, par laquelle le récit retrouve, de façon plus ou moins stéréotypée, l'inépuisable rhétorique des 'ajà'ib et gharâ'ib: mise en comparaison systématique des échelles; maximisation des écarts entre universalité des besoins et diversité des manières de les satisfaire; maximisation des effets de contrastes... Par ailleurs, ce dont nous fait témoins le déploiement de cette rhétorique, à travers les stéréotypes mêmes qu'elle égrène et les notations les plus convenues du point de vue de la tradition même de littérature de voyage " arabo-musulmane » qu'elle multiplie, c'est aussi de la progression de ce que l'on pourrait désigner comme la standardisation des normes mêmes du voyage et de leur alignement progressif sur la " qualité européenne » qui fournit, implicitement ou explicitement, les normes d'étalonnage, qu'il s'agisse de la qualité des hôtels, de l'agrément du voyage lui-même ou des «standards » de développement. J'y reviendrai dans la seconde partie de la présente étude. 


\section{Le voyage comme épreuve : normes et transgression}

17 À un deuxième pôle, qui tend à se renforcer, qualitativement et quantitativement au fur et à mesure qu'on se rapproche de la période contemporaine, le voyageur tend à s'affirmer de plus en plus vigoureusement comme le sujet de son voyage et, plus encore, de la relation qu'il en livre. Le paradigme est ici le voyage d'études, sans doute la variété la plus représentée, qu'il s'agisse de parcours réels ou romancés, dès le premier tiers de ce siècle, après le précédent magistral que l'on doit à Rifâ'a al-Tahtâwî. Sans abuser du modèle de la "quête initiatique ", force est bien de constater que bon nombre d'auteurs y ont eux-mêmes recours pour énoncer les enjeux de leur parcours : le voyageur se découvre lui-même à travers son voyage, et c'est le récit de cette expérience, parfois présentée comme une véritable rupture dans l'histoire individuelle de son héros, qui justifie le passage à l'écriture. Au risque même de se perdre: Adîb², sorte de double négatif de Taha Husayn, entame son périple par une double transgression - il ment en se présentant comme célibataire pour obtenir la bourse universitaire qui rendra possible son départ, et il est conduit par la logique de ce mensonge à répudier l'amour de jeunesse qui constitue la marque et le lien de son appartenance. Il sera dès lors libre de comprendre dans sa vérité même la civilisation et la culture de l'Occident: la musique, la littérature, l'art, la pensée délivrée de toute contrainte. Il devient un romantique. Mais c'est cette fusion même qui constitue sa perte morale et identitaire : il ne pourra plus faire retour et se perdra dans la folie et la dépravation faute d'avoir pu se raccrocher à son identité d'emblée sacrifiée au mirage de "l'aventure occidentale $»^{3}$.

18 Plus loin, ce qui justifie le recours au modèle de la quête initiatique, c'est la façon dont la présence insistante du voyageur à son voyage et au récit qu'il en fait tend à mettre à l'épreuve le système de normes et la "culture » dont celui-ci est porteur en même temps que la relation qu'il entretient à sa propre société. Mise à l'épreuve "négative ", d'une part: la découverte de l'Occident révèle pour ce qu'elle est l'amère réalité des contemporains « natifs » du voyageur. Mise à l'épreuve positive, d'autre part : le voyage met au défi le voyageur d'intégrer à sa propre ' vision du monde le fait qu'autrui quelle que soit par ailleurs la façon de le nommer et la position du voyageur par rapport à cette réalité - existe comme producteur du sens de son histoire propre voire, de plus en plus irrésistiblement, comme producteur du sens de l'histoire planétaire, y compris celle du Soi.

19 Je reviendrai sur cet effet de mise en interface des normes en la personne même du voyageur pour souligner ici la façon dont ce réinvestissement de son voyage par le voyageur autorise un considérable élargissement tout à la fois de son vécu en tant qu'enjeu de narration et de la représentabilité de l'altérité elle-même. La contrepartie de la présence du voyageur à son voyage est la valorisation de l'anecdote, parfois jusqu'au trivial, et la personnification/individualisation de l'Autre, parfois jusqu'à la caricature. Elle est aussi que la rihla cesse d'être ce parcours manichéen où le héros va d'affirmation de soi en affirmation de soi, prenant toujours le pas sur l'adversité et imposant à l'autre le respect, pour s'ouvrir à la prise en compte des vicissitudes du voyage et aux affres de l'exil, non plus sur le mode convenu de la «nostalgie pour le pays natal» (al-hanîn ila-l-awtân), mais avec la conscience que cette angoisse peut constituer un effet «objectif» de la mise en présence de l'Autre et du constat de sa "supériorité ». On peut faire ici l'hypothèse que ce qui constitue tout à la fois la 
«formule » et le principal enjeu de cette réinsertion du voyageur dans son voyage, c'est la relation même qu'il admet d'entretenir avec l'autre -, interactions qui, par leurs contenus et leurs modalités, donnent sens et valeur au voyage lui-même et permettent d'en tirer les enseignements. En d'autres termes, plus la personnalisation du voyage est grande, plus la présence de l'altérité se fait insistante en même temps que la perception de sa diversité, plus la question des normes est posée avec acuité, mettant en quelque sorte le voyageur devant ses responsabilités identitaires. La gestion du voyage - et de son écriture - engage ici la personne même du voyageur dans ses différents aspects, et en particulier moraux et politiques.

Sous bénéfice d'inventaire, on peut identifier deux cas de figure inverses, entre lesquels toutes les transitions - toutes les transactions - sont possibles, mais qui se présentent comme structurellement homologues pour ce qui est du rapport à l'expérience vécue : ce que l'on pourrait désigner, schématiquement d'une part, comme la levée - au moins provisoire ou partielle - des interdits, et, d'autre part, comme le refuge dans l'ascétisme des normes. Dans les deux cas, la mise à l'épreuve du voyageur passe par la proximité à la transgression, qui établit en sa personne même une sorte de ligne de démarcation entre identité et altérité: accepter les occasions ou les prétextes de transgression que vous offre l'autre, c'est en quelque manière lui rendre les armes et s'assimiler, ne serait-ce que provisoirement, à lui, accepter son empreinte. Sayyid Qutb (1951) en séjour d'études aux États-Unis en 1948-49, décrit crûment la séduction corporelle de

la jeune Américaine qui sait très précisément où celle-ci se situe. Dans son visage : des yeux rieurs, une bouche gourmande... Dans son corps: une poitrine ronde, des hanches pleines, des cuisses charnues, des jambes lisses... Et tout cela elle ne le cache pas mais elle le met en valeur. Parle vêtement: couleurs criantes qui éveillent les sensations les plus primitives, et coupes qui soulignent les formes du corps - et elles sont souvent, en Amérique, particulièrement ravageuses (sâ'iqa)! À quoi s'ajoute un rire provocant, des regards audacieux, des gestes osés, dont elle a conscience à chaque instant.

21 Mais c'est pour en conclure qu'il ne s'agit là que d'une séduction purement animale et qu'y céder serait indigne du respect qu'il se doit à lui-même. Quant à Louis 'Awad (1965, p. 50) lui aussi égyptien, lui aussi en voyage d'étude, il n'a rien de plus pressé, sur le bateau qui le conduit d'Alexandrie à Marseille, sur le chemin de Londres, que de se laisser entraîner par l'un des marins dans une «virée » dans les quartiers chauds de Gènes, lors d'une escale, au prétexte qu'

Il fait partie de ces gens toujours prêts à faire n'importe quoi, dans les limites du raisonnable (fî hudûd al-ma'qûl), pour apprendre quelque chose de nouveau ou pour voir quelque chose de nouveau - une disposition psychologique (hâla nafsiyya) dont Dieu protège [le lecteur].

L'expédition sera un fiasco pour L. 'Awad, lâché par son cicérone et qui ne trouvera son salut que dans la fuite devant l'agressivité commerciale des péripatéticiennes génoises, mais qui n'hésite pas - et c'est là ce qui m'apparaît comme le plus significatif - à se tourner lui-même en ridicule dans la narration qu'il donne de cet épisode. À ce niveau, l'important n'est pas tant de savoir si le voyageur a ou non transgressé, que de connaître le terrain ou l'idiome sur et dans lesquels s'énoncent les enjeux de la transgression elle-même. Une interrogation d'autant plus cruciale qu'une sorte d'implicite ou de ligne de fuite semble tramer ces rihiât "personnalisées ", où l'autre est pris en compte dans sa diversité : en caricaturant, ce qui fait, pour le voyageur, objet de possible transgression se situe aussi au plus près de la vérité de l'Autre. 
Versant «levée des interdits ", l'affirmation par le voyageur de son statut de sujet de son voyage peut être l'occasion d'un véritable dépouillement identitaire qui annonce la disponibilité de celui-ci à toutes les expériences. Après avoir noté le caractère convenu, théâtral de la scène de l'adieu à la mère-patrie -

Adieu, Égypte! objet de tout mon amour et de tous mes espoirs... Adieu, Égypte... J'ai beaucoup lu dans les romans ou les magazines, à propos de l'adieu au pays, aux parents, aux êtres chers... Une scène théâtrale toute prête... Son interprétation dépendait de moi...

L'Awad met en scène ce que Paul Fussel (1980) propose d'analyser comme des « rituels de passage » : à peine embarqué sur le bateau qui le conduira à Marseille, il se dépouille de ses vêtements « égyptiens » et revêt une veste « Norfolk » et un pantalon de golf,

parce que j'avais dans l'idée que les gens, en Angleterre, ont l'habitude de s'habiller de cette façon en voyage, sans que je sache très bien d'où me venait cette idée, de mes lectures ou du cinéma.

De fait, une fois en Europe - et même si la transgression peut apparaître moindre pour le chrétien qu'il est -, il arrivera à L. 'Awad de boire plus que de raison, d'entretenir des relations sentimentales «illicites ", voire de s'encanailler, convaincu d'être là au plus près de la vérité de son voyage, comme au cours de cette promenade au Quartier latin où il décide qu'il lui faut, à lui aussi, boire de l'absinthe,

pour oublier les vingt-deux années de mon existence qui s'étaient écoulées en vain entre le jour de ma naissance dans le village de Shârûna, district de Maghâgha, et celui de mon embarquement à bord du Kawthar à destination de Marseille... Pour faire comme Baudelaire, Verlaine, Rimbaud, Oscar Wilde et les autres «décadents» de la littérature, je devais vivre ne serait-ce qu'une heure dans l'ambiance dans laquelle ils avaient vécu... Comment pouvais-je laisser passer une occasion pareille?

Le voyage se présente ici comme la réalisation d'une anticipation constitutive de son projet même : faire des études et/ou jouir enfin de la permissivité qui est, comme le souligne Jean-Noël Ferrié, l'un des principaux traits stéréotypiques des mœurs sexuelles de l'Autre.

Le ton est tout autre, l'on s'en doute, chez un Sayyid Qutb, parangon de ce que j'ai désigné comme "l'ascétisme des normes ». Chez lui, la proximité de la transgression transgression du Commandement religieux, mais aussi transgression de l'humanité elle-même, proximité recherchée, de son aveu même - est celle de l'entomologiste (moins la neutralité) et a surtout fonction d'entretenir son indignation: pour se convaincre de la primitivité (bidâ'iyya), voire de la bestialité (wahshiyya) de la civilisation et du tempérament américains, il n'est que de suivre un match de football américain - sport qui n'a, dit-il, de commun avec le football que le nom -, ou un combat de boxe ou de catch et d'observer

l'attitude des foules qui suivent de tels spectacles [....] avec le même type d'intérêt que le public américain manifeste aux luttes entre bandes (jamâ'ât) et sectes (tawâ'if), entre peuples et nations, si bien qu'on ne comprend pas comment a pu être accréditée, notamment en Orient, le mythe (khurâfa) d'une Amérique éprise de paix.

Ce que lui dévoile la fréquentation, que l'on devine assidue et attentive, de l'Amérique profonde - au point qu'il prend soin de devenir membre d'un «club paroissial » dans chacune des villes où il réside, ce dont il ne retire pas une très haute idée de la religiosité américaine ou du rôle des églises dans la société -, c'est le fait que l'Amérique elle-même est fondée sur la guerre et le conflit - guerre des premiers groupes de colons entre eux, guerre d'extermination contre les Indiens, guerre de l'élément anglo-saxon contre l'élément latin, guerre d'Indépendance, de Sécession... -, 
qu'elle ne manquera pas d'étendre au reste du monde pour faire advenir le règne de l'Homme blanc (al-rajulal-abyad). "La troisième guerre mondiale est proche!", s'exclame-t-il. Ce qu'il me paraitt important de souligner ici, c'est qu'à ce stade de son évolution intellectuelle et politique, l'Amérique n'est pas le produit de l'Homme blanc, si par celui-ci on désigne la civilisation occidentale, à laquelle S. Qutb multiplie, par ailleurs, les références à connotation positives, en particulier à propos de l'Art ou de la Littérature, en tant que patrimoine de valeurs spirituelles et morales de l'humanité, dont lui-même revendique, implicitement, la maîtrise des codes : il s'est un temps laissé abuser, rapporte-t-il, par l'engouement extraordinaire des Américains pour l'opéra et le ballet dans la mesure où on peut «ne pas désespérer (d'une société) où le public est réceptif aux beaux-arts et aux émotions esthétiques ", mais il ne tarde pas à déchanter - ils ne s'y rendent que pour des raisons sociales, pour pouvoir en parler dans les réunions mondaines et se faire valoir en société! Plutôt, c'est bien l'Homme blanc luimême qui est le produit de l'Amérique comme parachèvement de la colonisation du monde, selon une logique identitaire que l'on pourrait qualifier d'extrémiste, par laquelle celui-ci entreprend d'imposer au resté du monde son hégémonie qui est celle de la matière et de l'organisation, seul apport des Américains au patrimoine de l'humanité :

Un peuple qui a atteint, dans l'univers de la science et du travail, le sommet du progrès et du développement alors qu'il reste primitif dans l'univers des sentiments et des comportements, et qui n'a pas dépassé sur ces terrains les premiers stades de l'humanité.

Le point crucial, c'est que le système de référents et de normes que mobilise $\mathrm{S}$. Qutb dans ses textes de 1951, peu de temps après son retour d'Amérique - il n'en sera plus de même dans les pages du Fî Dhilâl al-Qur'ân consacrées à son expérience américaine -, n'est que très implicitement et très secondairement le référent religieux, alors même que c'est bien son identité de musulman qui est exposée à la transgression, comme celle de ces «jeunes mécréants arabes" (shayâtîn al-shubbân al-'arab) qui étudient dans les universités américaines, et dont l'un avait été surnommé Abu al-Atâhiya dans le cercle de $S$. Qutb à cause de sa conduite dissolue. Et c'est l'humanité - non le caractère musulman, au moins explicitement - de leur société que mettent en péril ceux qui se font les avocats et les instruments de l'expansion de l'Homme blanc et qui,

ne trouvant rien qui vaille dans leurs personnes insignifiantes en rajoutent dans la glorification de l'Europe et de l'Amérique dans l'espoir d'en dériver un peu de valeur personnelle.

\section{Refondations identitaires du monde}

Les travaux consacrés aux rihlât ont surtout pris en compte, jusqu'à présent, les voyages à destinations européennes ${ }^{4}$. On peut se demander en quoi le développement, dès le début de ce siècle de voyages hors de l'espace européen restreint - vers l'est européen, les Amériques, le Japon et ce que l'on s'est habitué à désigner comme le "Tiers Monde », est de nature à permettre de reconsidérer les questions que l'on s'était habitué à poser à ces textes, articulées par le problème axiologique du rapport à la "modernité ", et de la relation entre celle-ci et l'« occidentalisation ». D'où le projet de tenter de saisir ce que j'ai désigné comme le "mode de présence du voyageur à son voyage » du point de vue du passage entre plusieurs mondes, entendus tout à la lois comme des configurations spatiotemporelles isolables - que celles-ci s'énoncent en termes géographiques, culturels, politiques, religieux... -, et dans l'acceptation qu'en 
ont Luc Boltanski et Laurent Thévenot, d'articulations institutionnalisées et hiérarchisées des gestions du lien social. De ce double point de vue, les questions auxquelles une relecture des rihlât permettrait de répondre ont trait à la façon dont l'altérité peut être instrumentalisée dans la production par les voyageurs d'univers de sens dans lesquels l'enjeu est rien moins que d'inscrire ce que je pourrais désigner comme des « projets d'être-au-monde ", individuels ou collectifs.

\section{Recentrements du monde}

31 En première lecture, les auteurs de rihlât sembleraient vouloir donner raison au partipris que je qualifierai d'« orientaliste », qui fait de l'Europe - de l'Occident -une sorte de «centre du monde », et du voyage en Europe, le voyage par excellence : d'une part, force est de constater que les rihlât européennes sont qualitativement plus différenciées, en particulier sur le terrain fictionnel; de l'autre, l'Europe figure concrètement, au moins jusqu'aux années cinquante, plus même que le centre du monde, la «porte » de celui-ci, voie d'accès d'autant plus incontournable, semble-t-il, que la destination est plus lointaine. Plus loin, c'est le monde lui-même qui, dans récriture des rihlât, tend à se constituer comme une sorte de projection de l'Occident hors de ses frontières. J'ai évoqué, à propos des guides de voyage, la façon dont les standards occidentaux fournissent les grilles d'étalonnage, par exemple en matière d'hôtellerie, d'organisation des transports et des services publics, de propreté ou d'hygiène. L'Europe - beaucoup plus encore que l'Amérique, sauf en matière d'organisation industrielle ou d'urbanisme - fournit le réfèrent de toutes les mises en comparaison et le principe de toutes les hiérarchisations. Plus loin, dans le contexte colonial et post-colonial, c'est dans la division des espaces eux-mêmes, en particulier des espaces urbains, que se donne à voir la projection de l'Occident hors de ses frontières géographiques, tout à la fois dans les corps et dans les esprits : vieilles villes, al-ahyâ' al-wataniyya, dit-on dans les années trente et quarante, ce qui désigne très précisément les quartiers «indigènes » versus villes nouvelles, occidentales, peuplées d'Occidentaux - de blancs... -, faites par eux et pour eux et où il arrive parfois que l'indigène lui-même ne soit pas admis ; secteurs économiques " mondialisés » articulés, dans les ports ou les "concessions ", comme à Shanghai, par les intérêts occidentaux versus marchés intérieurs, locaux, exotiques, qui servent parfois, il est vrai, de base d'appui à quelques commerçants chinois ou libanais, illustration du "génie » particulier de ces «races» pour le commerce; espaces de sociabilité modernisés/ occidentalisés - la presse, l'enseignement, l'armée, l'État lui-même - versus espaces de survivances, de blocage, de conservation du même, dans les campagnes ou les zones reculées ou au cœur même du lien social - la famille... Ajoutons que c'est dans les franges entre ces deux «mondes" que le crime et le vice trouvent leur terrain de prédilection et que prospère l'anomie, comme dans ces night-clubs de Shanghai, qui valent à cette ville le surnom de «Paris de l'Orient » - ou encore de «ville du démon » ou de « ville des passions » :

la contradiction [y] est à son comble entre l'ancien et le nouveau: la musique y est occidentale mais il s'y rencontre des Chinois revêtus de leurs robes qui ressemblent à des caftans, en train de boire de l'alcool et d'enlacer de jeunes Chinoises. Imagine-toi, ô lecteur, conclut l'auteur, le spectacle d'un cheikh enturbanné en train d'embrasser une jeune fille en se trémoussant. (Thâbit, 1936) 
Sans doute faut-il se résigner, à ce stade, à admettre que la saisie du monde que présente les rihlât est aussi et toujours, jusqu'au confins de la planète, une saisie de l'Occident et de la façon dont celui-ci est en train de reconfigurer le monde. Le mode de présence du voyageur à son voyage tend ainsi à devenir, dans la plupart de ces textes, mode de présence à l'Occident: le voyage s'inscrit dans un monde où - si l'on me pardonne cet anachronisme - la "globalisation» est à l'oeuvre: globalisation horizontale, coloniale et post-coloniale, qui fait que partout le voyageur rencontre l'Occident et ses œuvres; globalisation verticale, qui tend à diviser chaque société d'avec elle-même. Pour autant, la question n'est pas tant de savoir si les auteurs de nos rihlât sont " pour » ou « contre » l'occidentalisation du monde, que celle de savoir dans quels termes s'énoncent ces prises de position et comment elles se distribuent. Les remarques qui précèdent suggèrent une hypothèse classificatoire fondée sur la manière dont peuvent être articulés les deux termes de la quête en quelque manière que constitue toute rihla- Soi et l'Autre -, non pas tant pour montrer en quoi ils s'opposent ou peuvent être conciliés, que pour montrer comment se distribue la valeur elle-même - ce qui constitue la leçon du voyage - et comment elle circule entre ces deux pôles. Au risque de forcer un peu le trait, on peut, à la lecture de ces textes, dessiner un système à quatre positions symétriques pour ce qui est de la façon dont se structure la relation entre identité et altérité, inverses, pour ce qui est de la valence attribuée à l'un et l'autre terme.

\section{Amîr Buqtur : réalignement identitaire sur la modernité en marche}

33 Un premier cas de figure pourrait être décrit comme celui de l'acculturation identitaire: la distance - civilisationnelle, matérielle, morale, politique... - entre Soi et l'Autre, que le voyage permet de mesurer, apparaît telle qu'elle ne laisse pas d'autre choix que l'identification au modèle qu'il propose (qu'il impose), seule susceptible de rendre possible une accélération de l'histoire dans les territoires du Soi et de lui permettre de rattraper le train de la modernité en marche. Amîr Buqtur (s.d.) $)^{5}$ est le type même du voyageur " collectif ", " dépersonnalisé " ${ }^{6}:$ sa rihla est le voyage pédagogique d'un futur pédagogue, véritable apprentissage de l'Amérique qui a pris en charge sa formation dès avant l'aventure du voyage, qu'il présente comme une rupture radicale dans le cours de sa propre existence. Ce que donne à voir A. Buqtur à son lecteur, c'est d'emblée l'Amérique comme totalité, le "système » américain à l'œuvre dans tous les aspects et tous les registres des pratiques sociales et qui a fait de ce pays

le berceau de la civilisation moderne (al-madaniyya al-hadîthaj et le maître du monde.

L'Amérique, pour A. Buqtur, c'est tout à la fois «le pays de l'argent, de l'or et de la fortune » que chacun peut espérer conquérir pour peu qu'il retrousse ses manches et ne soit pas avare de sa peine, et

le seul pays au monde où s'appliquent les principes de la démocratie véritable,

dans une relation de causalité circulaire : c'est parce que le système politique américain est démocratique que chacun peut espérer un jour devenir riche, et c'est parce que le système social autorise et même encourage l'enrichissement individuel que la démocratie est nécessaire pour préserver l'égalité des citoyens. Sans que l'auteur nous explique de façon très convaincante comment ces deux aspects peuvent tenir ensemble si ce n'est en invoquant des "qualités " typiquement américaines - optimisme, pragmatisme, sens de l'intérêt général... -, produites par les conditions mêmes de la 
conquête de l'Amérique contre la nature sauvage - mais, après tout, Tocqueville luimême n'avait pas dit autre chose -, et qui font de la démocratie américaine une "démocratie sociale » avant d'être une "démocratie politique » comme en Europe. Sur ces bases, A. Buqtur va passer en revue l'une après l'autre, avec une systématicité appliquée, toutes les entrées du répertoire des stéréotypes des "miracles» de la modernité en marche, identifiant, après celles de l'Antiquité et du moyen âge, les « sept merveilles des temps modernes $»^{7}$. En bon pédagogue, il s'attache en particulier à décrire les différents instruments de socialisation qui entretiennent et développent ces "qualités américaines", en particulier l'école, mais aussi la presse, les musées, les associations..., dont les objectifs et le fonctionnement sont passés en revue avec une profusion de détails. Quant au Soi - à l'exception, comme on le verra, de la part prise par certaines communautés arabes, en particulier syriennes, au « rêve américain »-, il n'est convoqué dans ces pages que sur le mode du contraste négatif, du constat de la distance ou du manque, et même s'il prend la précaution d'affirmer, du bout des lèvres, son respect de la diversité des cultures, A. Buqtur ne cache pas vers où le portent ses inclinations :

Les Occidentaux sont plus réservés (akthar hishmatan) que les Orientaux. Ils préferent les ambiances calmes et quant ils parlent, ils évitent de hausser la voix pour ne pas déranger les autres. Jusque dans leurs mariages et dans leurs enterrements on n'entend pas une voix plus haute que l'autre. Quant aux Orientaux, ils ne savent parler qu'en criant et en haussant la voix. Leurs mariages ne sont que tambours et cymbales et si l'un de leurs proches meurt, ils emplissent l'univers de pleurs et de lamentations. Quand nous parlons, c'est toujours avec de grands gestes, ce qui est considéré, chez eux, comme une habitude détestable, contre laquelle je mets en garde tous ceux d'entre vous qui voyagent [dans les pays occidentaux].

On pourrait suivre encore longtemps A. Buqtur dans ce catalogue des différences significatives où il laisse au lecteur le soin de choisir ce qui lui semble le plus propice à la civilisation. Mais pourquoi faut-il qu'avec une constance qui ne doit sans doute rien au hasard, il ne cesse de clore ses démonstrations de la supériorité américaine par des exemples « limites », caricaturaux, contre-productifs, pourrait-on dire ? Qu'on en juge : les millionnaires sont tellement nombreux en Amérique, explique-t-il, qu'il a fallu inventer le mot «multimillionnaire» pour les différencier, mais même ainsi on ne saurait en préciser le nombre, si bien que,

pour (aire saisir au lecteur le grand nombre de millionnaires, je mentionnerai le fait qu'en 1922, 73 d'entre eux se sont suicidés!

37 Ailleurs, il note que la loi est si respectueuse des droits des individus que dans certains États les condamnés à mort peuvent choisir la façon dont ils seront exécutés. Ailleurs encore, il illustre l'importance du rôle joué par les femmes, en particulier dans la gestion des politiques sociales et éducatives, par l'exemple d'une inspectrice de l'Éducation, qui utilise son savoir-faire politique et son éloquence pour faire échouer une réforme des manuels scolaires, ruinant par là trois mois de travail de l'équipe pédagogique chargée de cette réforme pour le seul plaisir de marquer son pouvoir. On peut trouver la clé de ces «décrochements» dans la façon dont A. Buqtur traite des communautés syriennes qui ont réussi à passer au travers du " tamis » (gharbala) d'Ellis Island, «creuset où s'opère, écrit-il, la mutation du fer étranger en or américain ». Notons que c'est dans le chapitre qu'il consacre aux Syriens de Brooklyn que l'auteur expose sa propre représentation de la sélectivité du melting pot américain :

L'Amérique est, de tous les pays du monde, celui qui prête le plus d'attention aux peuples étrangers qui veulent pénétrer sur son territoire [...]. Ils veulent que les différents éléments dont se compose leur république soient l'élite des gens, les plus vifs de corps et d'esprit, ceux 
qui comprennent le mieux les principes de la démocratie véritable. Ils veulent empêcher complètement l'entrée des peuples jaunes et noirs car leur présence inciterait au mélange et au métissage, ce qui aurait pour effet de, faire disparaître la peau blanche attirante en même temps que l'élément saxon septentrional réputé pour son intelligence, son calme, sa maîtrise de soi et la qualité de son travail. [...] L'Amérique, ajoute-t-il, a appris à tester de nombreux aspects de la psychologie des peuples et des races qui se présentent à elle.

Pour leur chance,

les Syriens font partie des peuples que l'Amérique accueille volontiers, ou, au moins qu'elle ne rejette pas ou ne refuse pas d'accepter,

et qui, souligne-t-il, constituent un modèle d'acclimatation: d'une part, ils se conforment scrupuleusement aux habitudes, valeurs, comportements américains, de l'autre, ils vivent dans leur exil comme s'ils étaient dans leur propre pays, en quoi, précise A. Buqtur,

ils méritent véritablement la qualification de gentlemen.

Être soi chez l'Autre - prétexte pour l'auteur à exalter « les aspects de la grandeur orientale dans le Nouveau Monde » (madhâhiral-'adhâma al-sharqiyya fi-l-'âlam al-jadîd) : des quartiers entiers où l'on entend partout parler arabe et où les enseignes sont en arabe et en anglais, des cafés aussi beaux que ceux du Caire d'où s'échappent les voix enchanteresses d'AI-Minyalâwi ou de Munira al-Mahdiyya, et où même

les enfants avec qui nous jouions, parlaient l'arabe avec une pointe d'accent anglais, ce qui ajoutait encore à la beauté de cette langue.

1 Jusqu'aux Américains eux-mêmes qui apprécient de venir manger dans les restaurants orientaux, boire du café turc et écouter du 'ûd. Mais la leçon des Syriens de Brooklyn n'est pas seulement dans la réussite de leur acclimatation en Amérique ou dans leur capacité à se montrer les dignes héritiers des commerçants phéniciens, mais dans la façon dont ils font retour sur l'identité elle-même. Non pas seulement être soi chez l'Autre, mais être plus soi chez l'Autre qu'on ne l'est chez soi :

Je me prends à penser que les Syriens en Amérique sont différents des Syriens en Égypte: alors même qu'ils sont pleinement intégrés à la vie américaine, ils conservent soigneusement leurs habitudes orientales dans le vêtement, l'alimentation, le mobilier. En Égypte, les Syriens parlent français plus qu'arabe. Il sont français plus qu'arabe sur le plan de la langue et occidentaux plus qu'orientaux pour ce qui est des habitudes ('âdât). En Amérique on sent que le Syrien apparaît oriental au premier regard (tash'ur bi-anna al-Sûrî sharqî fî nadhar al-râl li awwal wihla); quant au Syrien en Égypte, il est difficile de le distinguer du Grec, duFrançais ou de l'Italien.

La véritable leçon des Syriens de Brooklyn, c'est bien qu'ils sont en Amérique ce qu'ils ne sont plus chez eux et qui trouve à s'épanouir dans les formes de la madaniyya américaine. Pour celle-ci, en retour, ils sont en quelque sorte un "supplément d'âme » et il ne fait pas de doute qu'A. Buqtur pense que l'Amérique en aurait bien besoin : à Brooklyn, écrit-il «l'Orient et l'Occident se rencontrent». Et il n'est pas indifférent pour le propos de la présente étude que ce soit une jeune Japonaise qui, sur le bateau du retour, tire pour lui la leçon de son voyage :

J'aime l'Amérique comme mon fiancé, dit-elle, mais j'aime le Japon comme ma mère.

\section{Muhammad Thâbit : la preuve par le Japon}

La rihla yabâniyya est un thème récurrent dans la littérature de voyage, à la fois par son traitement même et par les leçons qu'elle suggère ${ }^{8}$ : celui du Japon, du Meiji à nos jours, saisi comme le modèle emblématique d'une culture restée « authentique » mais qui a su 
se doter de tous les attributs de la modernité occidentale au terme d'un transfert réussi - l'élève ayant dépassé le maître sur son propre terrain -, démontrant par là ce que l'on pourrait désigner comme «l'appropriabilité » universelle de la modernité occidentale, dès lors définitivement promue au rang de patrimoine collectif de l'humanité. On peut sans doute voir dans le développement de cette thématique une réponse et une alternative à la «tentation occidentale » dont j'ai fait d'A. Buqtur une sorte de porteparole. L'identité, le Soi, vont ici être désignés comme les réceptacles nécessaires de la modernité, avec le Japon pour preuve de la viabilité du projet. Au prix, encore une fois, d'une reconnaissance : c'est bien toujours de l'appropriation de la modernité occidentale qu'il s'agit, même si l'identité va être convoquée pour rendre celle-ci possible et effective.

Rendant compte de son périple asiatique - qui le conduit successivement en Inde, au Japon et en Chine -, du point de vue d'un

regard égyptien, oriental [...] dont l'objectif est d'identifier (istiqrâ') les éléments de leur éveil(nuhûd) ou de leur stagnation (qu'ûd) dans l'espoir que nous nous inspirions de leurs méthodes confirmées (tarâ'iqiha al-muwaffaqa) et que nous prénions en compte les revers qu'il ont subis de manière à nous prémunir contre les difficultés qui menacent les nations à l'aube de leur éveil et aux différents stades de leur évolution-,

Muhammad Thâbit entreprend une de ces relations de voyage consacrées au pays du Soleil levant où ce dernier est saisi dans un double système de différenciation et de mise en opposition. Par rapport à l'Occident, d'une part: le Japon est le seul pays d'Asie, et peut-être du monde, qui se soit opposé victorieusement à l'intrusion de celuici et qui ait su se doter d'une civilisation, madaniyya, non seulement «viable » mais concurrentielle par rapport aux modèles européen ou américain.

En quelle langue rendre compte de la grandeur, de la maîtrise organisationnelle, de la sûreté de goût d'une civilisation qui manifeste dans tous ses aspects la majesté et la puissance de son souverain et qui surpasse, de mon point de vue, la plupart des capitales européennes. Les Japonais se voient ainsi créditer de toute une série de qualités positives, totalisées par l'exceptionnel attachement qu'ils portent à leur pays et qui les distinguent des Occidentaux :

Il ne fait pas de doute, écrit M. Thâbit, que les Japonais sont, beaucoup plus que les chrétiens, attentifs aux autres et prêts à se sacrifier pour l'intérêt général et qu'ils sont de tempérament tolérant et réceptif à la beauté divine de la nature. Quant aux Européens, ils croient, en théorie [en celle-ci], mais il la renient dans la pratique. Certains pensent que le Japon est le seul pays qui conjoigne la pureté et la beauté: on y rencontre l'amour du beau à un niveau de goût raffiné en même temps que la fidélité au groupe ('ashîra) s'accompagne du sens de la responsabilité sociale.

M. Thâbit ira jusqu'à nier la réalité de l'impérialisme nippon, ou à tout le moins à le justifier au nom de la «mission civilisatrice» du Japon, réfutant les accusations des Occidentaux contre ce pays - bellicisme (al-nuz'a al-harbiyya), matérialisme (hubb almâdda), absence de scrupules (al-tajarrud 'an al-damir) -comme "dénuées de fondement ». Différenciation, opposition aussi, par rapport au Soi : même si l'auteur affirme que

les étrangers ne comprennent pas le système social du Japon, que nous autres, Égyptiens, sommes en situation de mieux comprendre dans sa vérité $d u$ fait de sa proximité à notre propre système,

le Japon reste le pays où le voyageur doit s'attendre à se retrouver dans les situations ' les plus incompréhensibles, voire les plus choquantes. M. Thâbit nous fait ainsi part de sa gêne quand, dans une auberge où il est descendu, une jeune fille le dépouille de sa 
veste pour lui faire enfiler un kimono, gêne qui tourne à la confusion lorsque d'autres jeunes filles prétendent l'assister dans sa toilette et qu'il plonge dans le baquet d'eau chaude pour soustraire sa nudité à leur regard, polluant celle-ci et privant de toilette les autres clients de l'hôtel. Il découvre ainsi que le spectacle de corps nus n'est pas une honte pour les Japonais, ce dont il conclut sobrement que

ce que nous considérons comme blâmable est pour eux naturel.

Un sentiment d'«étrangéité » maximale encore renforcé par le fait qu'au-delà de l'extrême politesse des Japonais, ils restent difficiles à connaître vraiment: ils n'acceptent pas d'étrangers comme amis et

le Japonais, quand il parle avec un étranger, se souvient toujours qu'il représente le Japon,

ce que l'auteur explique par une faible individualisation (du'f al-istiqlâl al-dhâtî), qui est également ce qui permet de rendre compte de la faiblesse de la créativité personnelle il n'y a pas de grands intellectuels ni de grands artistes au Japon - et l'absence totale de sens de l'humour des Japonais (iftiqâr al-yabânîff tafâhum al-fukâha wa-l-mazâj).

51 Dans l'inventaire des lieux communs ou des stéréotypes sur le Japon que les rihlât contribuent à mettre en place, on ne s'étonnera pas de l'attention consacrée par M. Thâbit à la condition féminine au Japon, l'occasion pour lui de maximiser les écarts. À commencer bien sûr par les geishas,

ces accompagnatrices professionnelles (samîrât muhtarif âtj, formées dans des écoles spéciales, sans lesquelles aucune réunion ne saurait se tenir.

52 M. Thâbit ne se méprend pas sur le statut des geishas dans ces réunions et sur leur fonction purement « décorative ", ni sur le statut «dominé » de la femme japonaise les Japonais sont intimement persuadés, souligne-t-il, que les hommes sont supérieurs aux femmes et ne « considèrent pas, comme les Européens, que le beau sexe (al-jins allatif) soit l'âme d'une soirée ou sa raison d'être (hayât al-majâlis wa rûhuha) -, mais ce qui le choque, c'est qu'ils paraissent, dans leur misogynie, estimer plus l'immoralité des femmes que leur vertu : d'une part,

ils n'accordent pas autant d'importance que nous à la virginité et à l'honneur ('ird)

53 et les jeunes filles non mariées jouissent d'une liberté quasi totale ; de l'autre, et alors même que la femme mariée se doit d'être d'une fidélité irréprochable,

leur respect de la femme immorale (al-'âhirât) est aussi grand, sinon plus que leur respect de la femme mariée, si bien qu'en cas de besoin, par exemple pour payer une dette, c'est le père qui pousse sa fille vers la prostitution (da'âra) dans la mesure où il s'agit de protéger la famille en tant que pilier de la nation (rukn qawmî) influant sur la stabilité de l'État et de la patrie (...), un comportement qui peut nous sembler sauvage et barbare (hamajî wa wahshî) mais qu'ils justifient au nom du devoir d'obéissance des enfants aux parents et de l'impératif de les sortir de leurs difficultés. Le comble étant que les jeunes gens rivalisent pour épouser de telles femmes, une logique que nos esprits sont bien incapables de saisir.

54 On pourrait multiplier à l'envi les situations où le voyageur expérimente et donne à voir l'altérité maximale de la société japonaise - panthéisme shintoïste, sacralisation de l'empereur, sens de l'honneur exacerbé qui pousse les Japonais au suicide pour les raisons les plus futiles... L'important est que cette altérité maximale n'interdit pas d'affirmer une proximité en quelque manière qui fait que la transplantation pure et simple de l'« expérience » japonaise dans le territoire du Soi est non seulement possible mais hautement désirable :

le Japon est le pays le plus proche de nous par bien des aspects, que n'y envoyons-nous des groupes d'étudiants et de commerçants pour étudier les moyens économiques et moraux qui lui ont permis de réaliser ce surprenant progrès? 

peut voir une sorte de condensé des leçons tirées par les rihlât japonaises. Jusqu'à suggérer l'hypothèse que la maximisation de la distance entre le Soi et l'altérité constitue le principal ressort à l'œuvre dans la production discursive du «modèle japonais ", à quoi concourent ces rihlât. Au-delà des différences irréductibles, emblématiques, qui les opposent, ce qui constitue la proximité essentielle de Japon et de l'Égypte - de l'Orient arabe - c'est le projet identitaire lui-même, celui d'inscrire la modernité dans une identité réaménagée. De ce point de vue, la véritable leçon du Japon, ce n'est pas tant ou pas seulement qu'une modernisation « réussie » peut et doit s'opérer dans un cadre identitaire :

Les fondements de la civilisation demeurent, dans ce pays purement oriental car le Japon ne croit pas à la philosophie de l'Occident, à ses moeurs, à ses usages sociaux, à sa religion ou à sa politique; les gens conservent leurs maisons, leur façon de se vêtir, leurs temples et leurs jeux (...), comme si le Japon voulait respecter son présent et son passé à la fois et comme s'il voulait que l'Occident le respecte en tant qu'État puissant sans renoncer à sa personnalité passée.

C'est aussi l'idée selon laquelle le renforcement de l'identité est la condition nécessaire d'une accélération $6 e$ la marche à la modernité. C'est bien parce que le Japon s'est retranché dans son identité - aussi « exotique » puisse-t-elle apparaitre au lecteur de M. Thâbit qu'il a pu se permettre de construire sa modernité sur des bases aussi « hétéroclites ». La sagesse du Japon, nous explique-t-il, est de ne pas s'être reposé sur un seul État pour importer les éléments de son progrès : l'organisation de l'armée était inspirée du modèle français jusqu'à la défaite des forces françaises contre l'Allemagne ; le modèle de la flotte est britannique, tout comme les chemins de fer; le système financier est américain; le système politique est allemand, l'Allemagne étant le pays auquel le Japon a le plus emprunté "parce que, de tous les pays, c'est celui qui ressemble le plus au Japon. » Mais la synthèse est indubitablement japonaise, et c'est elle qui constitue la leçon du voyage : l'objectif de l'instruction depuis la période du Meiji est, souligne M. Thâbit d'

acquérir (naql) la civilisation matérielle de l'Occident pour conserver la nature (kiyân) de l'État, le Japon n'admettant pas que sa civilisation et son système social soient inférieurs à ceux de l'Occident, si bien que le principal souci des dirigeants nationalistes (al-zu'amâ' alwataniyyîn) a été de faire la synthèse (jam') entre ces deux composantes en dépit de leur antipathie réciproque (tanâfur).

Dans cette formulation, ce qui fait la spécificité de l'«exception japonaise » est précisément ce qui autorise la transposition de la leçon qu'il donne aux «nations renaissantes » dans les termes mêmes de la politique du Soi :

Alors que c'est la renaissance industrielle qui, en Europe, a influé sur les systèmes politiques, c'est l'inverse qui s'est produit au Japon où la révolution (inqilâb) industrielle a été le produit direct d'un changement de système politique. C'est l'État qui a ouvert les usines et qui continue à les diriger; c'est lui qui a envoyé des étudiants à l'étranger pour y étudier l'industrie et le commerce; c'est lui qui a fait venir des experts étrangers, qui a créé des écoles gouvernementales, des chambres de commerce...

Ce sont là les termes mêmes du projet de renaissance initié en Égypte par Muhammad 'Alî et Ismâ'îl, et M. Thâbit ne se prive pas de pousser quasiment terme à terme la mise en parallèle : en Égypte comme au Japon, l'industrialisation n'a pas remis en cause le socle fondamentalement paysan de la société, ce qui se traduit dans la structure du commerce extérieur - exportation de matières premières agricoles, importation de produits manufacturés - "semblable à celle qui prévaut chez nous"; en Egypte, 
comme au Japon, le démarrage d'une industrie nationale n'a pu se produire qu'au terme d'une partie de bras de fer avec les Occidentaux à propos des protections douanières, visant à remettre en cause les « capitulations " consenties à ces intérêts à d'autres époques; en Égypte comme au Japon, la croissance de la population apparaît comme un problème crucial dont la solution réside dans « l'absorption de la croissance démographique par le développement de l'industrie ». D'où cette question que ne cesse de réitérer $\mathrm{M}$. Thâbit au fur et à mesure que progresse sa mise en comparaison :

Qu'attendent donc nos gouvernants pour appliquer le plan de développement industriel mis en œuvre par le Japon et qui correspond (tulâ'im) à notre situation?

La proximité des situations et des problèmes est ici ce qui permet d'annuler la maximisation des différences tout en lui assignant son statut discursif : la garantie, en quelque sorte que les Égyptiens ne deviendront pas Japonais alors qu'ils sont en lutte pour ne pas devenir Occidentaux!

\section{Louis 'Awad : le travail de deuil de l'identité}

60 boursier égyptien à Cambridge à la veille de la deuxième guerre mondiale, sans doute le plus «personnel » des voyageurs dont il a été question dans le cadre de la présente étude. Ce qui singularise son écriture et en constitue la dimension proprement subversive, c'est, je l'ai suggéré, sa façon de ne pas se présenter systématiquement à son avantage, voire de ne pas hésiter à se tourner lui-même en ridicule - Don Quichotte de Minya (son terroir d'origine), se brocardant lui-même, suite à une consommation excessive d'alcool qui l'a rendu malade et lui laisse une "vraie gueule de bois américaine » (sudâ'amrikâni asît). Ce que nous donne à lire L. 'Awad, c'est la façon dont se met en place, dans des espaces structurés par le lien colonial - tout comme aussi par la montée des nationalismes -, une pratique du voyage en quelque sorte routinisée par la densification des échanges, en même temps que la façon dont l'hypothèse même du départ est dorénavant inscrite dans les imaginaires. C'est aussi la façon dont l'expérience de l'altérité, le vécu même du voyage permettent de faire retour sur le Soi et d'engager le travail de deuil de l'identité.

'Awad inscrit d'emblée la subversion au cœur même de la rihla, dans la langue même qui en livre la leçon, avec le recours au dialecte "intégral " ${ }^{9}$ par quoi il poursuit un objectif qui a directement à voir avec la pédagogie du voyage : lever un obstacle entre le lecteur et la réalité décrite et rendre compte des états d'âme du voyageur dans la langue même dans laquelle ils sont vécus. Il y a là plus qu'un effet de réalisme, la recherche d'une efficace propre de l'écriture qui permette au voyageur de formuler le sens même de son voyage :

Jusqu'à aujourd'hui, je ne sais pas dans quel style (uslûb) écrire. Si je te dis je suis entré dans tel musée et j'ai vu telle ou telle chose, il ny a plus de différence entre moi et Muhammad Thâbit et tu as meilleur compte à acheter le Guide bleu. Mais si je me mets à te parler des rêves qui habitent mon coeur, si je te fais entendre le son des cloches et si je te montre mes châteaux en Espagne, tu diras que ce ne sont que mes rêves...

Quant à la pédagogie même du voyage, elle tient dans la circularité du regard qui fait passer le voyageur de la vérification d'une image "antécédente ", " préconçue » de l'Occident - de ses stéréotypes, si l'on veut -, dont tout un chacun est dorénavant porteur, grâce notamment aux relations de voyage, à la réévaluation «objective » des performances du Soi, y compris dans le cours du voyage lui-même, et qui constitue la 
véritable leçon de celui-ci. Au point de départ de son voyage, L. 'Awad place le désir de "vérifier la carte postale":

Avant même de quitter l'Égypte, je connaissais déjà Londres quartier par quartier, mais seulement à partir des livres et des récits: Westminster, Picadilly, Soho, Hyde Park étaient à leur place dans la carte de mon esprit... Imagine-toi ce que cela peut faire de rêver chaque nuit à une chose et de l'avoir soudain devant les yeux... Il y a bien de quoi être ému ! et politique : j'ai évoqué, dans ce qui précède, ses émois au Quartier latin à l'idée de marcher sur les traces de Baudelaire, Verlaine et des autres... En fait, par formation et par inclination, son univers littéraire est plutôt anglophone - Byron, Shelley, Oscar Wilde - à la différence de son univers politique, marqué par la Révolution française. En bon romantique qu'il est, cette vérification ne peut être qu'édifiante : la France est bien le pays des Lumières tout comme l'Angleterre est celui de la Magna Carta et de I'Habeas Corpus -même si lui apparaît aussitôt le revers de la médaille : toutes deux sont des empires coloniaux où sont bafoués au jour le jour les principes mêmes sur lesquels sont supposés se fonder leurs systèmes politiques et où le racisme ordinaire est au fondement des relations sociales ${ }^{10}$. Le parcours dans l'espace, pour « vérifier la carte postale ", est ce qui le confronte à l'Histoire elle-même en tant que processus pourvu d'un sens - par exemple quand on lui montre l'endroit où fut assassiné Alexandre de Yougoslavie :

Je me mis à méditer sur cet événement et sur la manière dont il avait pu se produire. Ce sont des choses qui marquent. J'avais la sensation que je "voyais" l'histoire, un sentiment qui m'est venu en Europe mais qui ne m'était jamais venu en Égypte. l'Histoire, voire, de son aveu même, de prendre pied dans la réalité: jusque-là, explique-t-il, si on lui demandait à quelle époque il aurait choisi de vivre, il répondait à celle de la Révolution française,
pour lire Rousseau alors que sa pensée était encore brûlante, voire Marie-Antoinette manger ses gâteaux, entendre Mirabeau, assister à la prestation de serment du jeu de paume, écrire à la craie sur les murs des Tuileries, suivre Charlotte Corday dans la salle de bain de Marat et enfin assister à l'essor de l'aventurier corse [...] mais si on me reposait aujourd'hui la même question, je choisirai de naître au $\mathrm{XX}^{e}$ siècle dans la mesure où j'ai réalisé que nous vivons la période la plus cruciale (akhtar al-marâhil) par laquelle soit passée notre espèce depuis qu'elle a pris conscience d'elle-même, entre marche vers la civilisation et course vers la catastrophe.

65 L'avènement de la deuxième guerre mondiale, qui abrège son séjour en Angleterre ne tardera pas à mettre cette deuxième éventualité à l'ordre du jour, dramatisant la leçon du voyage : le fascisme, entr'aperçu en Italie, la persécution des juifs en Allemagne, la brutalité des sociétés occidentales pour leurs propres laissés-pour-compte, et toujours le racisme, mais qui prend une dimension nouvelle.

Jusque-là, l'Europe qu'il nous avait décrite était surtout représentée comme le nouveau «terrain d'aventure » et d'expérimentation des jeunes Égyptiens qui, comme l'auteur lui-même, avaient l'opportunité de s'échapper de la mère-patrie, par exemple au prétexte d'un voyage d'étude pris plus ou moins sérieusement : espace de déploiement de shilal -groupes de fraternités électives, si l'on veut - qui se font et qui se défont, traçant tout une géographie de cafés

- du moment qu'il y a des Égyptiens dans un pays où pourraient-ils se retrouver sinon dans les cafés? -, 
de lieux de rencontre et d'espace de transgression. Version "édifiante », c'est Muhammad Mandhûr, père putatif de la critique littéraire égyptienne moderne, lui faisant découvrir le Paris secret, celui des initiés. Version transgressive, c'est ce groupe qui se retrouve tous les jours au premier étage du café Dupont, au Quartier latin, pour jouer au poker jusqu'à la fermeture et finir la nuit en buvant et en fumant du haschisch avant de recommencer le jour suivant et le suivant, ou encore le «club des Pharaons ", à Cambridge, vitrine officielle des Égyptiens dans cette ville, mais dont la principale activité semblait être de se réunir tous les quelques jours pour acheter en commun

un tonneau de bière ou un carton de gin ou de whisky, ou quelques bouteilles de vin français ou allemand et discuter sans fin à propos de mille sujets (...) ou encore d'aller draguer les filles dans les boites de nuit.

Le point crucial est que cette prise en compte du vécu réel du voyage est ce qui amorce le retournement du regard. Rendus à eux-mêmes par la mécanique du voyage et les possibles qu'il ouvre, les jeunes Égyptiens sont en France ou en Angleterre ceux-là mêmes qu'ils sont en Égypte : des frustrés
dont on ne sait pas bien s'il faut se fâcher contre eux ou les plaindre, malheureuses victimes d'une neurasthénie sexuelle qui grignote leur raison et éclate sur leur peau comme des boutons [...]. Quant à la classe des effendis, en Égypte, ce n'est pas une classe, poursuit-il, mais une collection de complexes psychologiques (majmû'a min al-'uqad al-nafsiyya) dont on ne peut espérer venir à bout sans une nouvelle orientation sexuelle (tawjîh jinsî jadîd) fondée sur une liberté utilisée de façon modérée et une compréhension plus saine des choses.

D'une part, c'est cette constitution psychologique qui permet de comprendre pourquoi, pour bon nombre d'entre eux, ils passent littéralement à côté de leur voyage : ils ne comprennent même pas, quand ils en ont, les codes qui président au comportement de leurs petites amies, ce qui les conduit à relayer à leur tour le mythe de l'« immoralité » occidentale en se vantant de conquêtes souvent imaginaires. De l'autre, la comparaison avec la façon dont vont les choses dans les sociétés occidentales permet, sinon de percevoir les dysfonctionnements de la société égyptienne, du moins de replacer ceuxci dans de nouveaux systèmes de causalité : en Europe,

il n'y a pas de poussière. C'est bien cela : il n'y a pas de poussière. Dans aucun des pays du Tout-Puissant, il n'y a de poussière! Pourquoi faut-il que l'Égypte seulement en soit envahie? Il y a de quoi se poser des questions. Serions-nous les seuls à avoir une campagne? Mais tous les pays du monde ont des campagnes. Ou peut-être est-ce parce que Mr Macadam faisait partie des ennemis de l'Orient que le ministère égyptien des Travaux publics a décidé de ne pas goudronner les routes... (...) Poussière dans les dossiers du gouvernement. Poussière dans les yeux des gens. Poussière partout! Je comprends bien pourquoi la galabiyya du paysan, pourquoi sa maison sont crasseuses: la pauvreté. Ce que je ne comprends pas, c'est pourquoi nos routes et nos rues sont comme les a créées la nature. Le gouvernement égyptien $n$ 'est pas pauvre qui dépense chaque année 15 millions de livres pour entretenir ses fonctionnaires...

70 Ne nous y trompons pas : ce qui se joue à travers ce type de formulations - et elles sont nombreuses dans la rihla de L. 'Awad - c'est bien une "désidentitarisation» des problèmes posés dans sa propre société. La leçon de l'Europe, c'est bien, en définitive, que l'Égypte n'est pas vouée par nature à son arriération et que des solutions existent, inspirées ou non des modèles occidentaux. Plus positivement, la leçon du voyage, c'est, pour L. 'Awad, la relativisation des différences et des identités elles-mêmes. D'une part, il n'y a pas quelque chose qui serait "l'Egypte est une partie de l'Europe », comme 
l'avait rêvé Ismâ'îl - non que l'Egypte en serait "indigne", mais parce que cette proposition traduit une ignorance des réalités européennes elles-mêmes :

Les différences entre l'Angleterre et la France, ou entre les Anglais et les Français sont plus fondamentales (jawhariyya) que celles entre la France et l'Égypte ou entre les Français et les Égyptiens.

De l'autre, il n'existe pas d'identités, aussi différentes puissent-elles apparaître, entre lesquelles il ne soit pas possible d'établir des correspondances : l'animation du Quartier latin, la nuit, ne fait-elle pas penser à celle de Sayyida Zaynab - et réciproquement - ? Et Marius et Olive ne sont-ils pas l'équivalent de Goha?

Qu'on ne s'étonne pas, donc, si je dis que les Marseillais et les Alexandrins ont plus d'une chose en commun,

à commencer par le fait d'habiter sur les deux rives de la même mer. En fait, ce n'est pas tant la Méditerranée que découvre L. 'Awad - lui-même, à la fois "sa'îlî̀ et "british", était bien peu méditerranéen - que le voyage comme pratique de la connivence, par quoi le voyageur peut se sentir partout chez lui.

\section{Abdelmajid Benjelloun : l'identité comme manque}

C'est une rihla paradoxale qu'accomplit, dans l'entre-deux-guerres, le Marocain Abdelmajid Benjelloun (1992) : par son parcours en quelque sorte «inversé » - de Manchester où sa famille a tenté de s'établir, à Fès où il retrouve ses racines; et parce qu'il ne s'agit pas, comme pour les autres voyages, d'une projection du voyageur vers l'avenir et l'extériorité, mais d'une remontée rétrospective dans le temps et les territoires de l'enfance ${ }^{11}$. Plus encore que pour les autres textes évoqués dans ce qui précède, l'enjeu du voyage est ici, explicitement, le retour, c'est-à-dire le moment où devront être intégrées à la représentation du Soi les leçons de celui-ci. Ce qui incite à prendre pour point d'accroche de notre lecture de ce texte, le point d'aboutissement du parcours d'A. Benjelloun, qui est lui-même un nouveau départ, rendu possible par l'achèvement d'un processus tragique de marquage des espaces entre lesquels l'enfance de l'auteur a été déchirée. Ce processus constitue la leçon même du voyage, celle que livre le récit de

la vie d'un enfant qui a vécu entre deux milieux presque contradictoires - un cas fréquent,

mais relaté par un nombre infime de personnes.

74 Arrivé au terme de son enfance et du voyage qui le ramène vers ses racines, $\mathrm{A}$. Benjelloun est devenu un nationaliste, et c'est ce qui va le remettre en marche à destination de l'Égypte. Mais cette fois, il s'agit d'un exil, non d'une émigration: absence volontaire au territoire du Soi, à la fois pour se soustraire à l'anomie de la situation coloniale qui prévaut au Maroc, qui a mis en péril jusqu'à sa santé mentale, et pour entamer le combat de la reconquête. De ce point de vue, ce que l'auteur entreprend, en livrant le sens de son parcours éclaté, c'est d'énoncer le besoin tragique d'une identité, faute de quoi on est condamné à errer sans fin entre deux rives expérience personnelle d'un enfant

envahi du sentiment effrayant qu'[il] était né pour être étranger : au pays d'où nous venions, les gens me regardaient comme un étranger, et au pays qui était soi-disant le mien, c'était pareil-,

mais aussi, métaphore de la domination coloniale. 
76 L'on ne s'étonnera pas de ce que l'espace britannique, où se situe le point de départ du parcours d'A. Benjelloun soit saisi et représenté de façon paradoxale par le tout jeune enfant qu'il est à l'époque, même s'il s'agit là de perceptions très largement « reconstruites » par l'adulte écrivant. D'une part, l'Angleterre est décrite comme le lieu d'un séjour provisoire, peut-être précaire - ce qui se confirmera -, mais en même temps valorisé, à la fois lieu de peur et de bonheur. Un espace dans lequel on vit sur le mode de l'extraterritorialité : à la fois entouré par un monde qui vous tient pour étranger et qui peut se montrer hostile, et où l'on vit sur le mode du repli sur soi. L'Angleterre est aussi ce monde où peut être reproduit le partage entre un espace domestique, espace de la famille dont les relations avec son environnement sont en quelque sorte "médiatisées" par une famille amie elle-même "atypique ", grécoanglaise, et l'espace publique, espace d'affirmation des hommes :

Les activités de ces Marocains émigrés étaient commerciales; je n'y entendais rien et ne leur attribuais, dans ma tendre enfance, que peu d'importance. Je peux dire aujourd'hui que tous respiraient le bien-être et la gaieté, signes évidents de la réussite [...] ils tenaient aussi beaucoup à jouir de la vie, cette vie agréable que leur offraient les villes et villégiatures anglaises.

77 D'autre part, l'Angleterre elle-même existe par rapport à autre espace originel, un pays lointain d'où, affirmait notre gouvernante, nous étions venus; un pays où tout est fabuleux.

Plus loin :

Ce mot de Maroc évoquait en moi une foule d'images, tirées des histoires entendues, et se confondait dans mon esprit avec le pays des Nègres, pays des créatures chez qui tout est bizarre : dimensions, couleurs, faits et gestes.

C'est aussi l'espace de ses peurs et de ses cauchemars d'enfant: petit, il n'ose pas regarder les servantes noires dont il a

une crainte profonde, y voyant les modèles vivants de ces créatures monstrueuses et cruelles des contes de Maman.

80 À l'inverse, toute beauté est pour lui d'Angleterre et il oppose les maquillages chargés et les couleurs criardes qu'affectionnent les femmes de son entourage à la gracieuse simplicité des amies anglaises de sa famille :

Elles égalaient Angie en beauté; mais pourquoi celle-ci était-elle séduisante, auréolée de bonheur et de joie? Pourquoi, au contraire des Marocaines, était-elle gracieuse, élégante? Tout en elle était beau et attirant. Les autres, je le voyais bien, étaient belles mais pas élégantes, et cela était dû à leur costume, de mauvais goût et sans grâce. Au lieu des cheveux ondulés d'Angie, je voyais des tas de soieries et de bijoux qui cachaient les têtes. Au lieu du corps délié d'Angie, je voyais des vêtements pendants comme des rideaux; au lieu de la silhouette gracieuse d'Angie, je ne voyais rien du tout; au lieu du visage net et épanoui d'Angie, je voyais des faces barbouillées de teintures, et je les plaignais.

81 À partir de ce territoire de l'Autre devenu paradoxalement sien, ne serait-ce que parce qu'il n'a pas souvenance d'autre chose, ce sont en fait deux rihlât que va accomplir A. Benjelloun dans le territoire également paradoxal d'une identité étrangère ou du Soi vécu comme altérité, deux voyages aux enjeux radicalement différents. Une première fois, c'est comme «touriste », pourrait-on dire qu'il s'y rend, pour faire la connaissance de sa famille restée au pays, à une période où le retour définitif n'est en aucune façon à l'ordre du jour- un voyage qu'il aborde avec quelque inquiétude

vers un pays redoutable, que mon père connaissait et où il ne risquait donc rien mais où nous - un jeune parent et lui-même -, étrangers à ce pays, étions exposés à ce qu'il nous arrive malheur ${ }^{12}$. 
mais qui va se transformer en source d'émerveillement. Ce qui fait la spécificité de ce premier parcours, c'est que les deux termes en sont également valorisés, encore que pour des raisons différentes, voire contradictoires : d'une part la réussite de son père à l'étranger, sa propre "étrangéité » de " petit Anglais » sont sources de gratification, comme lorsqu'il découvre le bonheur d'avoir un grand-père qui le regarde avec des yeux qui semblent dire

comme les temps changent! Ils ont fait de toi un Européen, mon petit, mais ce n'est pas grave, approche, je te montrerai comment être un bon Marocain ;

de l'autre, une fois oubliée la première impression de délabrement, il découvre dans la maison familiale de Fès

une vie nouvelle, toute de plaisir, qui charmait vraiment les sens et me dégoûtait de la vie dans la noire Manchester... Je passai à Fès six semaines, pensant qu'aucune autre ville au monde ne pouvait m'offrir une vie aussi belle. Je jouissais de tout: le bonheur, la joie, l'entrain qui se lisaient sur le visage des enfants, et ces choses magnifiques et étonnantes $q u$ 'on trouvait dans les marchés. J'avais si longtemps entendu les histoires de Maman quand elle me parlait du Maroc jje ne pensais pas que la réalité puisse surpasser le rêve jusqu'au moment où j'arrivais dans le pays.

84 Malgré la chaleur de ces retrouvailles, ce premier voyage apparaît comme une parenthèse qui ne saurait remettre en cause le projet de vie en Angleterre pour luimême ou pour sa famille:

J'avais connu le Maroc sous tous ses aspects pendant les six semaines passées là-bas, et y avait trouvé bien des sujets d'émerveillement;je pensais même que le globe terrestre manquait largement des agréments existant au Maroc; mais malgré tout, je ne souhaitais pas y vivre toute ma vie. J'imaginais d'y retourner en touriste mais il ne me vint pas à l'idée que j'y reviendrai définitivement.

De retour en Angleterre, une fois retombées les émotions suscitées par le voyage, le rapport qu'il fera à ses petits camarades, très satisfait de se faire ainsi valoir à leurs yeux, prend pour objet le "pays du bizarre » : un pays où les gens se mettent en groupe pour faire leur toilette, où les femmes mettent leurs plus beaux habits pour rester chez elles et les cachent pour sortir et où, pour chaque chose

Il y a une manière spéciale, par exemple la coupe des cheveux chez eux: ils ont du poil comme nous, sur la tête et au menton; nous, nous nous rasons le menton mais pas la tête; mais, eux, ils se rasent la tête et pas le menton; ils ont le menton sur la tête et la tête au menton.

Mais même ainsi, il n'en reste pas moins qu'un contenu positif a été donné par ce voyage à sa propre altérité dans son environnement britannique, et qu'il découvre la façon de la valoriser aux yeux de l'Autre britannique - le mystère exotique - et à ses propres yeux - un « supplément d'être ».

87 C'est cette belle construction qui va être mise à bas par le second voyage qui le ramène au Maroc, définitivement cette fois. Les affaires de son père ayant périclité, comme celles de tous les Marocains établis à Manchester dont les activités ne s'appuyaient pas sur des connaissances ou une vision globale des choses, indispensables pour garder le cap en temps de crise,

88 l'environnement britannique devient franchement inhospitalier et l'alternative du retour au pays apparaît dès lors sous un jour positif, l'occasion de

mettre un terme à cette ombre où nous avions passé huit ans. C'était le moment, la boucle allait nous ramener à la blanche patrie bien-aimée et nous délivrer de Manchester la noire, la détestée.

Égypte/Monde arabe, 30-31 | 1997 
eirs ne tarderont pas à être déçus et le Maroc va se muer, pour A. Benjelloun en un espace de déperdition accélérée du sens. Tout ce qui lui avait paru enchanteur lors de son premier séjour devient marqueur d'une sorte d'exil intérieur, dont l'amertume emportera sa sœur, de santé fragile. On commence par le revêtir d'habits marocains qu'il découvre ne pas aimer, alors que, la première fois, il les avait trouvés " pittoresques",

des habits amples et flottants qui auraient suffi à habiller dix enfants de ma taille, habits qui gênaient mes mouvements, m'incitaient à la paresse et tuaient en mon corps tout dynamisme, carcans pour l'esprit et fers pour mon enfance, qui semblaient vouloir m'entraîner à toute vitesse vers la décrépitude.

Plus même, il lui faut à lui aussi se raser la tête et sacrifier cette chevelure qui révélait aux autres enfants qu'il était un "infidèle ». Il va d'échec scolaire en échec scolaire, dans des écoles où "aucun élève ne savait pourquoi ses parents l'envoyaient en classe ", sous la férule de maîtres ridicules et ignorants. Sa seule passion et la seule identité de rechange à laquelle il peut se raccrocher va être le football, au grand dam de sa famille, qui le sort de son milieu feutré et étriqué de petits notables rassis, et le rapproche des gamins de la rue. Mais le comble de la confusion identitaire est atteint avec son passage par la Qarawiyyîn qui lui donne "l'impression de traverser un immense asile d'aliénés » dans lequel pourtant il va s'engouffrer dans l'espoir de se recréer par là un ancrage :

Si je regrettais quelque chose, écrira-t-il à propos de cette expérience qui fera quand même de lui un écrivain raffiné de langue arabe, ce ne serait pas ces jours d'autrefois où tout mon idéal était de devenir un docteur au turban monumental. De quoi en effet avoir honte, si ce n'est d'avoir jadis, à la fleur de l'âge, aspiré à devenir un vieillard?

Lui qui jusque-là était relativement assidu dans l'accomplissement de la prière se met paradoxalement à la négliger :

Quand j'eus fait la connaissance avec la mosquée Qarawiyyîn, il arriva le contraire de ce qu'on pouvait attendre: mon observance de la prière à l'heure précise s'attiédit; puis je me permis des omissions partielles; enfin j'en arrivai à une négligence incroyable. Et vint un jour où mon père m'ordonna de la faire; je m'aspergeai d'un peu d'eau, entrai dans la pièce voisine et dis à haute voix les paroles de la prière allongé sur le matelas. Je faisais l'effort de la prière pratiquement sans prier.

Le constat opéré a posteriori de son impuissance à faire tenir ensemble les aspirations contradictoires de son enfance britannique et de son adolescence marocaine, impuissance qui prend la forme d'un trouble psychologique et somatique intense et donne lieu, de la part du narrateur à une réévaluation « objective » de l'« humanité marocaine ", entre négativité coloniale et exigences de réforme endogène, va être le point de départ de sa prise de conscience nationaliste en même temps que de sa vocation littéraire et de son identité d'intellectuel. La prise de distance par rapport à l'identité opérée par le voyage - identité magnifiée par l'absence, renvoyée au sordide de sa condition par la comparaison au moment du retour - désigne le caractère inéluctable de sa reconstruction. 


\section{BIBLIOGRAPHIE}

ABU-LUGHOD Ibrahim, 1963, Arab Rediscovery of Europe, a Study in Cultural Encounters, Princeton University Press.

AL-'ALAWI Saîd Bin Sa'îd, 1995, Urûbba (i mar'ât al-rihla : sûrat al-âkhar fi adab al-rihla almaghribiyya al-mu'âsira (L'Europe au miroir de la rihla : l'image de l'Autre dans la littérature de voyage marocaine contemporaine), Rabat, Université Mohammed V, Publications de la Faculté des Lettres et des Sciences humaines.

AL-'AMRAWI Idrîs Ibn Idrîs, Tuhfat al-malik al-'azlz bi-mamlakat Bâriz :

-1989, éd. et trad. MBAREK Zaki, La société française sous Napoléon III, Rabat, IURS.

-1992, trad. BARBULESCO Luc, Le paradis des femmes et l'enfer des chevaux, (préf. BEN ACHOUR Y.), Paris, Éditions de l'Aube.

'AWAD L, 1965, Mudhakkirât tâlib bilha, Le Caire, Al-kitâb al-dhahabî (Ouvrage réédité par Dâr alma'ârif, Le Caire, 1991).

BENJELLOUN A., 1992, Fi-l-tufûla, trad. GOUIN Francis, Enfance entre deux rives, Rabat, Wallada.

BUQTUR A., s.d., Al-dunya fiAmrîka, Le Caire, al-Matba'a al-'asriyya.

FUSSEL P., 1980, Abroad: British Literary Travelling Between the Wars, New York.

HUSAYN Taha, 1988, Adib, ou l'aventure occidentale, trad. TAHA HYSAYN Amina et Moënis, Paris, Éd. Clancier Guenaud.

KADDOURI Abdelmajid, 1995, Sufarâ' maghâriba fi Urûbba, 1610-1922 (Ambassadeurs marocains en Europe, 1610-1922), Rabat, Université Mohammed V, Publications de la Faculté des Lettres et des Sciences humaines.

LEWIS Bernard, 1982, The Muslim Discovery of Europe, New York, W. W. Norton \& Co. [traduction française, Comment l'Islam a découvert l'Europe, Paris, La Découverte, 1984].

LOUCA Anouar, 1970, Voyageurs et écrivains égyptiens en France au XIXe siècle, Paris, Didier.

AL-QÂDIRÎ Abu Bakr, 1982, Sittat Ayyâm fî-l-Yabân, Casablanca, Matba'at al-najâh al-jadîda.

QUTB Sayyid, 1951, « Amrîka allatî ra'aytu : fi mîzân al-qiyam al-insâniyya », Al-Risâla, XIXe année, vol. 2, n" 959, cité dans AL-KHÂLIDî Salâh 'Abd al-Fattâh, 1987, Amrika min al-dâkhil bimindhâr Sayyid Qutb, Jeddah, Dâr al-munâyara.

RASHSHÂD Bey, s.d., Siyâsa fi-l-Rûsya, Le Caire, Matba'a al-taqaddum (Texte publié en feuilleton dans Al-Mu'ayyiden 1914).

SHAFÎQ D., s.d., Rihlatîhawla al-'âlam, Le Caire, Dâr bint al-nîl.

THABIT Muhammad Thâbit, 1936, Jawla fî rubû' Asya bayn Misr wa-l-Yabân, Le Caire.

YÂRID Nâzik Sâba, 1992, Al-rahhâlûn al-'arab wa hadârat al-gharb fî al-nahda al-'arabiyya al-hadîtha (Les voyageurs arabes et la civilisation occidentale dans la renaissance arabe moderne), Beyrouth, Dâr Nawfal. 


\section{NOTES}

1. Le corpus de textes que je travaille pourrait être qualifié, en l'état actuel, d'« aléatoire ", dans la mesure où il ne comporte pas d'ambition d'exhaustivité, même s'il tend à s'étendre de plus en plus dans la perspective d'une étude plus systématique de ce thème qu'il me reste à entreprendre. Il se compose d'une cinquantaine de relations de voyage, (rihlât, sing.: rihla) égyptiennes (30) et marocaines (18), réelles ou romancées, diversement datées - entre 1830 et 1995. Par rihla, j'entends tout texte où le voyage hors du pays natal fournit prétexte et matière à l'écriture.

2. Adîlb, ou l'aventure occidentale, traduit de l'arabe par Amina et Moënis Taha Husayn, Paris, Éd. Clanciér Guenaud, 1988. T. Husayn s'attribue, de façon transparente, le rôle de l'ami - aveugle, fidèle mais raisonnable -, qui prend la pose du narrateur pour défendre, malgré ses frasques, la mémoire du héros et tirer la leçon de son périple.

3. Est-il hasardeux d'avancer que, dans toute la littérature romanesque de cette période, Il n'y a pas de retour heureux? Il y a peut-être là quelque chose de l'ordre du fil conducteur ou de l'hypothèse pour la lecture des "véritables" relations de voyage, celles en particulier qui affichent leur vocation pédagogique. Si la finalité du voyage est elle-même pédagogique, et si l'on peut y voir, comme le proposent de nombreux analystes, l'une des modalités « pragmatiques » de l'émergence de la figure moderne de l'intellectuel, ce qu'anticipent les romanciers, c'est l'incapacité des nouveaux acteurs produits parce parcours, "modernisés ", si l'on veut, à exercer leur magistère pédagogique dans le contexte - colonial, féodal, religieux... - de la société héritée.

4. Voir, par exemple : Abu-Lughod, 1963 ; Louca, 1970 ; Lewis, 1982 ; Yârid, 1992 ; Al-'Alawî, 1995 ; Kaddouri, 1995.

5. Amîr Buqtur, copte protestant formé dans la mouvance des missions américaines très actives en Égypte des les années vingt, sera le premier secrétaire général égyptien de l'Université américaine du Caire.

6. Les seuls épisodes véritablement personnels de son voyage sont son séjour forcé à Ellis Island, à la fois antichambre et anti-Amérique, où il se retrouve "par erreur » - suite à un défaut de transmission de ses documents d'inscription à l'université -, dont il se sent presque responsable, comme atteint par l'indignité de ceux qui ne sont pas admis sur le territoire américain; il oubliera très vite, nous dit-il, le sentiment de déshumanisation qu'il a ressenti au sein de l'humanité réprouvée d'Ellis Island, et même, malgré la cruauté de ces mesures, avouera sa compréhension pour l'attitude des Américains qui «exigent de leur gouvernement qu'il promulgue des lois pour mettre un terme à l'invasion (qui menace le pays) et interdire son territoire aux individus faibles de corps et d'esprit, de réputation ou de moeurs douteuses et à tous ceux qui risqueraient de salir la réputation du pays et de submerger l'élément saxon dont ils s'enorgueillissent. (Buqtur, s.d., p. 13), voir infra; le deuxième épisode personnel est une visite aux chutes du Niagara qui commence de façon bucolique mais manque de mal tourner à cause du froid, ce qui justifie le récit qui nous est fait de cet épisode.

7. Le télégraphe, la vapeur, l'automobile, l'avion, le radium, certains composés chimiques comme les anesthésiques et les désinfectants, l'analyse biologique et les rayons X, (Buqtur, s.d., p. 31).

8. Voir, entre autres exemples, Al-rihla al-yabàniyya li-sumuw al-amîr al-jalîl Muhammad 'Ali Pacha al-brins, shaqJq al-hadrat al-fakhîma al-khidiwiyya, 1910, Le Caire, al-Matba'a al-amîriyya ; Thâbit Muhammad (1936) ou encore le long chapitre que Mansûr Anîs consacre au Japon dans Hawla al-'âlam. Dernière occurrence en date, concernant le Maroc, les deux chroniques du "Bloc-notes " de Ben Jelloun Tahar dans La vie économique, 20 septembre 1996, sous le titre « Lettre du Japon ».

9. C'est-à-dire pour les dialogues mais également pour les parties narratives.

10. L. 'Awad fait ainsi part au lecteur de son trouble à être, en raison de sa complexion foncée, en permanence pris pour un Indien: "Chaque personne que je rencontre me demande si je suis 
Indien... Qu'est-ce que ça veut dire ? Il n'y a que les Indiens qui ont la peau sombre ? Je me mis à détester les Anglais parce qu'ils oubliaient complètement mon pays et à détester les Indiens parce qu'ils faisaient se tromper les Anglais sur le compte des Égyptiens !» ('Awad, 1965, p. 110).

11. Ce qui me fonde à traiter ce texte comme une rihla, c'est la façon dont l'auteur fait du passage d'une rive à l'autre l'objet même de l'écriture et s'opère par l'écriture l'articulation de ces deux espaces. Une fois le retour véritablement accompli - c'est-à-dire au moment où un nouveau départ sera devenu possible, on verra sur quelles bases le récit s'interrompt. Du point de vue qui est celui de la présente étude, on peut regretter que ce renoncement d'A. Benjelloun à poursuivre la rédaction d'une autobiographie nous prive du récit de son voyage et de son séjour dans l'Égypte des Officiers libres.

12. Il apprendra par la suite que le malheur spécifique qui attend son jeune parent est que son père a décidé de le marier au pays alors que lui-même veut épouser une Anglaise.

\section{INDEX}

Mots-clés : altérité, identité, voyage, voyageurs égyptiens

\section{AUTEUR}

ALAIN ROUSSILLON

CNRS, IRMC-Rabat 